\title{
Nordiques
}

$34 \mid 2017$

La mer Baltique comme zone-frontière : perspectives environnementales, géopolitiques, culturelles

\section{Quelle homogénéité baltique au regard des dynamiques maritimes et portuaires?}

Arnaud Serry

\section{(2) OpenEdition}

\section{Journals}

Édition électronique

URL : https://journals.openedition.org/nordiques/1739

DOI : $10.4000 /$ nordiques. 1739

ISSN : 2777-8479

Éditeur :

Association Norden, Bibliothèque de Caen la mer

Édition imprimée

Date de publication : 1 novembre 2017

Pagination : 79-98

ISBN : 9791095914006

ISSN : $1761-7677$

Référence électronique

Arnaud Serry, "Quelle homogénéité baltique au regard des dynamiques maritimes et portuaires ?», Nordiques [En ligne], 34 | 2017, mis en ligne le 15 octobre 2021, consulté le 27 novembre 2021. URL http://journals.openedition.org/nordiques/1739 ; DOI : https://doi.org/10.4000/nordiques.1739 


\section{Quelle homogénéité baltique au regard des dynamiques maritimes et portuaires?}

Arnaud Serry*

RÉSUMÉ

Héritière d'une configuration spécifique au regard de l'économie maritime mondiale, la mer Baltique est aujourd'hui, et depuis une vingtaine d'années, un espace maritime dynamique. La région connaît des taux de croissance de son trafic maritime parmi les plus élevés au monde ayant entraîné le renouveau de son monde portuaire. Toutefois, entre coopération, concurrence et dissensions, il convient de sinterroger sur ce qui caractérise aujourd'hui cet écosystème. Ce développement profite-t-il de la même manière à l'ensemble des entités portuaires, est-il appelé à perdurer notamment face à la volonté de le Russie de se recentrer sur ses ports et donc de renverser la logique géographique de cette région qui, depuis la création des ports hanséatiques, s'est développée principalement comme interface entre l'Europe du Nord-Ouest et son hinterland russe?

\section{ABSTRACT}

The Baltic Sea has been developing specific patterns in terms of maritime economy, and it has become in the last 20 years a dynamic maritime area. The region has some of the world's highest rates of growth in maritime shipping, which has brought intense transformations to its harbour facilities. However, this specific Baltic Sea system is still marked both by cooperation, competition, and quarrels amongst its main actors. Have all actors and especially all harbours beneficiated from the recent developments? Are these developments under threat from Russia's intention to refocus its action on its own harbours? Will this decision possibly reverse the geographical logic of a region that, since the creation of the Hanseatic ports, had developed as a connection between North-Eastern Europe and the Russian hinterland?

* Arnaud Serry est maître de conférences à l’université du Havre. 


\section{INTRODUCTION}

Penser les espaces riverains de la Baltique revient à s'interroger sur les évolutions extraordinaires qui ont affecté l'Europe au tournant des années 1990. La région baltique, changeante et diverse, est un exemple d'entre-deux géographique ${ }^{1}$. Elle a été successivement occupée par les chevaliers teutoniques, la Suède, la Prusse et la Russie tsariste. On y parle neuf langues différentes, les traditions religieuses varient d'un territoire à l'autre, les niveaux de développement économique sont relativement contrastés, certains États sont membres de l'Union européenne (UE) et d'autres non. Divisés au cours de la guerre froide, les espaces riverains de la mer Baltique sont longtemps restés des marges géographiques, bien loin des centralités continentales de l'Empire soviétique et des zones de croissance d'une Union européenne en pleine formation. Jusqu'à la chute du rideau de fer, la mer Baltique a été une frontière " chaude ", un glacis militaire traversé par le rideau de fer, elle est aujourd'hui devenue une mer ouverte à des échanges internationaux en croissance constante.

Nulle part ailleurs en Europe il n'y a eu, ces dernières années, autant de mutations politiques et économiques. L'éclatement du bloc de l'Est a, dans une certaine mesure, unifié cet espace. Le caractère maritime de cet espace est fréquemment cité comme révélateur de son unité, comme le résume très clairement Jacques Marcadon $^{2}$ : "Le trait d'union entre les pays de la Baltique est la mer. " Le rôle du transport maritime confère à la mer Baltique des particularismes régionaux, parmi lesquels l'idée qu'il contribuerait très fortement à une régionalisation. Les ports baltiques, pivots d'un réseau de transports - maritimes - s'intègrent dans un système plus vaste et créent du lien dans l'espace baltique. En effet, le développement portuaire et l'évolution des circulations maritimes sont révélatrices des mutations économiques et territoriales en raison de leurs interactions dans ce système marqué en même temps par la concurrence et la complémentarité.

Dans ce cadre, ce papier propose de dégager les spécificités de ce monde portuaire et maritime baltique. Au-delà d'une approche statistique, les dynamiques et les stratégies des différents acteurs sont au cœur de la réflexion. Dans un premier temps, sera abordé l'aspect de la Baltique comme trait d'union économique (et culturel). Dans une seconde partie, sera précisée la diversité des situations maritimes et portuaires baltiques en considérant les évolutions récentes, notamment en termes de trafic et de dynamiques. Enfin, la troisième partie s'intéressera aux facteurs de diversité et à leur développement sous l'œil de la marchandise et des acteurs du shipping.

1 Nicolas Escach, Arnaud Serry, "Les méditerranées ou l'émergence de régionalisations réticulaires ", in Géographie des mers et des océans, N. Escach (dir.), Malakoff, Dunod, 2015, p. 226-227.

2 Jacques Marcadon, "L'évolution récente de l'activité maritime et portuaire dans les pays de la Baltique ", Bulletin de l'Association de géographes français : la nouvelle Europe médiane, 1998, p. 428-436. 


\section{UN ESPACE MARITIME « COUTURE »}

Les caractéristiques physiques de la mer Baltique sont considérées comme les premiers facteurs d'unité tels que l'illustrent le rôle de l'englacement de la mer en hiver et ses conséquences économiques sur l'activité des ports ${ }^{3}$. Ce sentiment va même plus loin : ainsi, le critère de participation au Conseil des États de la mer Baltique (CBSS) est lui aussi physique puisque c'est celui des bassins des cours d'eau se jetant en Baltique. De ce point de vue, le concept de Méditerranée peut être appliqué à la Baltique qui en intègre trois caractéristiques majeures : des réseaux interdépendants intégrés principalement via des villes portuaires, des limites encore floues et son positionnement comme espace intermédiaire et carrefour. Cette régionalisation réticulaire est portée par l'organisation du transport maritime régional et lui apporte ainsi une véritable unité.

\subsection{L'enjeu du transit international}

Au cours des années 1990, le problème s'est posé d'une voie sûre et économiquement satisfaisante de sortie des produits russes vers l'Atlantique. Si la perméabilité des frontières post-soviétiques varie selon les lieux, elle dépend aussi de la nature des flux. Même si la Russie met régulièrement en œuvre une politique visant à instrumentaliser l'économie pour servir des buts politiques, les circulations à l'échelle du marché mondialisé traversent plus facilement les frontières que les flux d'échelle locale ou régionale. À cette fin, les solutions proposées ont notamment consisté à intensifier le réseau ferroviaire et routier régional, particulièrement les connexions vers la Finlande (un accord ferroviaire bilatéral de dérégulation a, par exemple, été signé en septembre 2016), mais la voie maritime demeure la plus intéressante et a imposé le recours aux infrastructures portuaires existantes. Les Baltes ont compris que leurs économies, au marché intérieur réduit, ne pouvaient se développer sans utiliser les réseaux hérités et les relations privilégiées tissées avec le voisin russe. L'espace baltique a donc vu renaître les circulations commerciales traditionnelles ${ }^{4}$. Les ports régionaux prêtent ainsi une attention particulière à la desserte de leur arrière-pays post-soviétique, où ils sont en concurrence entre eux et avec les ports russes du golfe de Finlande ${ }^{5}$, et interviennent pour restreindre les obstacles à la circulation.

3 Élise Lépy, Les glaces de mer en mer Baltique. Étude géographique et implications environnementales et sociétales à partir de l'étude comparée de la baie de Botnie (Oulu. Finlande) et du golfe de Riga (Lettonie), thèse de géographie, université de Caen, 2009, 420 p.

4 Arnaud Serry, "Les activités commerciales et marchandes à la frontière orientale de l'Union européenne : le cas de figure russo-balte ", Territoire en mouvement, no 29, 2016.

5 Saint-Pétersbourg est le port historique russe dans la région, complété par Vyborg et Vysotsk. La Russie a ouvert un port pétrolier à Primorsk en 2002 et entrepris depuis 2006 de développer le port d'Oust-Louga, aujourd'hui devenu le plus grand port de la Baltique en tonnage. 
Dans un second temps, la Fédération de Russie a débuté une stratégie d'autosuffisance portuaire et d'évitement par le développement de nouveaux ports de commerce dans le golfe de Finlande, la construction de conduites et l'abandon partiel de certains oléoducs sur les territoires baltes. L'exemple le plus marquant est en 2003 la fermeture pure et simple de la conduite reliant le port letton de Ventspils au réseau d'exportation russe de pétrole brut. En 2015, le transit de marchandises de la CEI représentait encore près de $40 \%$ du trafic du port lituanien de Klaipeda et près de $80 \%$ des trafics portuaires lettons ou estoniens. Les trains-blocs au départ des ports baltes circulent ainsi vers Minsk, Odessa, Moscou, Riga ou Almaty, impliquant notamment des facilités douanières.

Dans la pratique, les mobilités à l'échelle du marché mondialisé, en particulier celle des matières premières vers l'ouest et celle des produits manufacturés vers l'est, traversent facilement l'espace baltique dont elles constituent des éléments structurants. La fluidité de ces circulations commerciales met en évidence un phénomène de persistance des territoires : en effet, les acteurs économiques ont vite compris le bénéfice qu'ils pouvaient tirer de ces flux construits sur des partenariats historiques. Il est important de souligner que la logique est/ouest est prédominante $^{6}$ alors que les flux méridiens, aujourd'hui intra-européens, restent modestes. Par ailleurs, la discontinuité engendre ici des processus d'organisation spécifique de l'espace, un espace gateway, dans le sens de région polarisant des flux très importants avec un impact sur le territoire environnant. Les marchandises diffusent à partir de cette région gateway dans un milieu différent et avec des modes de transport différents.

\subsection{Trafics portuaires : la croissance en partage}

Héritier d'une configuration spécifique vis-à-vis de l'économie maritime mondiale, la mer Baltique est à l'écart des principales routes maritimes qui desservent les grands ports mondiaux. En Europe, ceux-ci s'organisent autour de la Northern Range ${ }^{7}$ et la Baltique est une façade maritime secondaire. Elle n'est en effet pas suffisamment attractive pour les escales directes des plus gros navires : situation en cul-de-sac et fermeture par les détroits danois, faiblesse des volumes, fragmentation des escales réparties sur des distances rapprochées en sont les principales causes. La Baltique est donc essentiellement desservie par un réseau de navires feeders ${ }^{8}$ qui se rattache indirectement au réseau maritime mondial via les grands ports européens. Qu'il s'agisse des ports de l'ouest ou de ceux des autres

6 Nicolas Escach, Arnaud Serry, "Les ports de la mer Baltique entre mondialisation des échanges et régionalisation réticulaire », in Géoconfluences, 2013, [http://geoconfluences.ens-lyon.fr/test/doc/transv/ Mobil/MobilScient7.html] (consulté le 3 octobre 2017).

7 Ensemble des ports s'étalant du Havre à Hambourg.

8 Nom donné à un navire de petit tonnage qui relie des ports où n'escale pas le navire mère. Il permet l'éclatement sur différents ports d'une cargaison apportée sur un port principal ou, inversement, de collecter les marchandises pour les regrouper sur le port principal. 
rives, les relations directes avec des ports extérieurs à l'Union européenne sont rares, voire inexistantes pour la majorité d'entre eux. La régionalisation baltique existe ainsi via l'agrégation des liaisons maritimes sur les ports de Hambourg et Bremerhaven. L'ouverture économique de l'espace baltique se manifeste paradoxalement par la rétraction de l'avant-pays de ses ports.

Nous sommes ici au cœur d'un positionnement périphérique, ne pouvant plus attendre de liaisons directes avec les avant-pays lointains, son intégration se fait sous le registre de la domination par des places extra-baltiques.

Parallèlement, depuis le milieu des années 1990, une croissance pérenne et soutenue du trafic, la modernisation et la restructuration portuaires ont été les traits majeurs de tous les ports de la Baltique. Cette croissance apparaît comme la principale caractéristique commune à la communauté portuaire baltique, que les ports soient nordiques, baltes ou russes. Le trafic global en mer Baltique a ainsi doublé de 1997 à 2016 passant de 420 millions de tonnes à près de 800 millions de tonnes, alors que dans le même temps la croissance du trafic maritime mondial, bien que rapide, n'était que d'environ $65 \%$. La quasi-totalité des ports baltiques a connu une augmentation de son trafic (voir fig. 1).

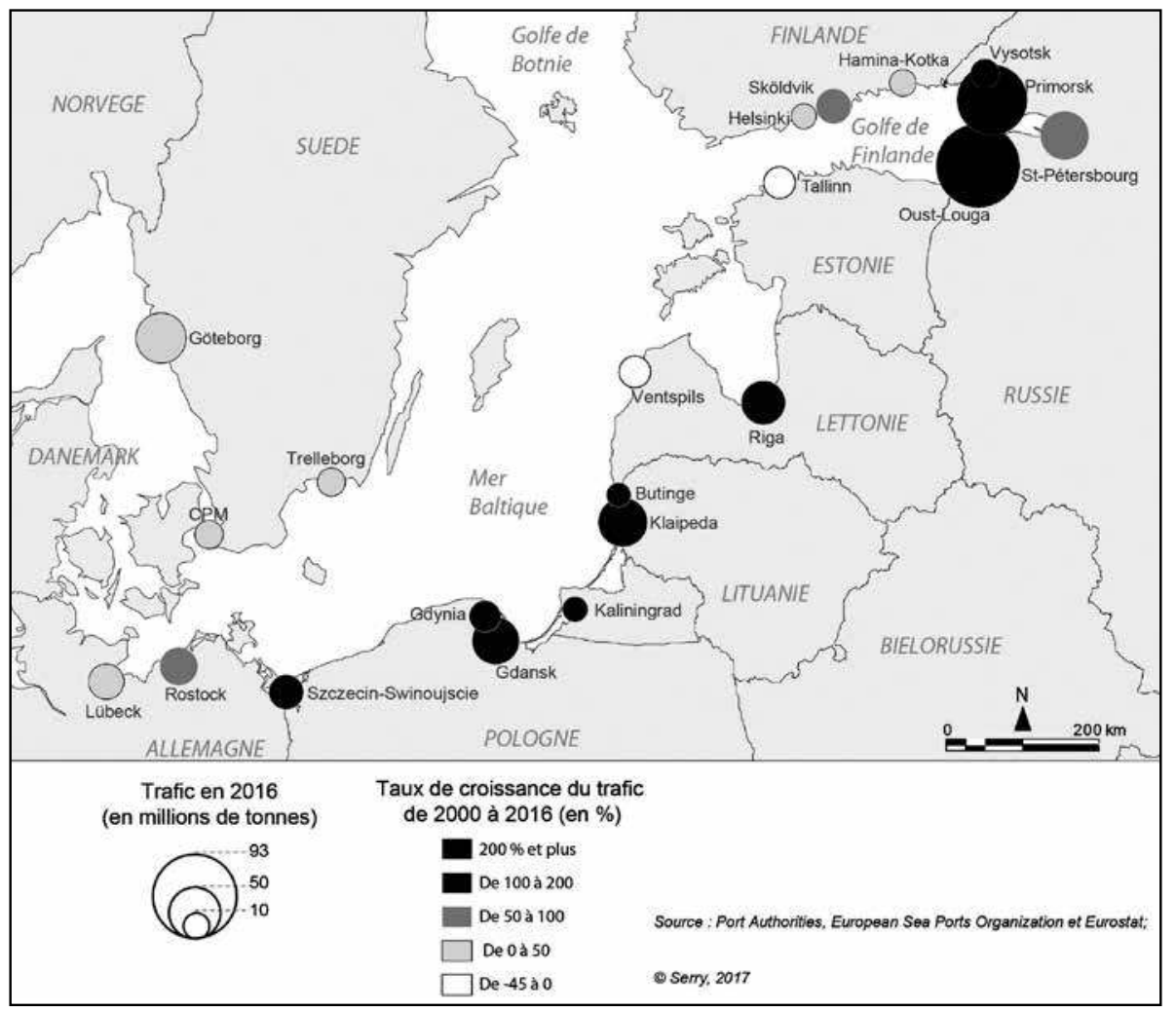

Figure 1 - Les 20 premiers ports de la Baltique en 2016 
Force est de constater que la Russie continue de jouer un rôle crucial dans ce développement des circulations maritimes. Les besoins vitaux de la Russie pour exporter ses matières premières, notamment ses hydrocarbures, en constituent en effet le premier facteur de croissance. Celui-ci est renforcé par la croissance économique mondiale et l'augmentation des volumes de marchandises transportés par voie maritime ainsi que par les profonds bouleversements géopolitiques de la région au début des années 1990 ayant ouvert la rive orientale à l'économie de marché et favorisé le développement de nouveaux trafics.

Simultanément à l'accroissement global du trafic, les flux maritimes ont connu une redistribution catégorielle au profit des hydrocarbures et du conteneur qui constituent désormais l'essentiel des tonnages manutentionnés, à côté de la spécificité roulière baltique. La Baltique est en conséquence dotée d'un excellent réseau pour le transport de passagers : environ trente millions de personnes voyagent chaque année par ferry. Cette mise en relation participe sûrement au développement régional, économique, mais aussi à l'intégration des différentes rives. La croissance générale de l'activité maritime dans la région illustre ce rôle de trait d'union qui est régulièrement assigné au transport maritime.

\subsection{Un espace de coopération maritime}

La mer Baltique a été un espace de coopération et de voisinage bien avant que l'UE ne cherche à y développer ses propres politiques extérieures régionales?. Le rapprochement le plus important dans le contexte général d'affrontement entre les deux blocs s'est effectué autour de la thématique environnementale. Signée en 1974 et révisée en 1992, la Convention pour la protection du milieu marin de la mer Baltique (convention d'Helsinki) est le premier texte-cadre d'envergure rédigé par les États riverains de la mer Baltique ${ }^{10}$. L'approche de la convention d'Helsinki fut novatrice, car elle avait pour ambition de discerner la problématique de ce milieu dans son ensemble : prévenir la pollution causée par les navires, la pollution d'origine terrestre et la pollution résultant de l'exploration du fond de la mer et de son sous-sol. Elle régit également la coopération visant à lutter contre la pollution marine par les hydrocarbures et autres substances nocives. La convention traduit la ferme détermination des parties contractantes à assurer la réhabilitation écologique de la Baltique et illustre le rapprochement qui s'est opéré entre les rives au cours des vingt dernières années ${ }^{11}$.

9 Anaïs Marin, «Argument baltique : faux prétexte et modèle juste », Outre-Terre, no 23, 2009, p. 347-362.

10 Nicolas Escach, Arnaud Serry, «Les méditerranées ou l'émergence de régionalisations réticulaires », op. cit.

11 Arnaud Serry, "Le transport maritime en mer Baltique, entre enjeu économique majeur et approche durable ", Revue d'études comparatives Est-Ouest, vol 44, nº 3, 2013, p. 89-123. 
Au niveau européen, la décennie 1990 a été marquée par des invitations à la coopération en Baltique (Conseil des États de la mer Baltique). Après l'élargissement de 2004, la Commission européenne a lancé le concept de macrorégion pour définir un ensemble d'États ou de régions associés autour d'un ou plusieurs caractères et défis communs ${ }^{12}$. Pour la région baltique, la première stratégie européenne macro-régionale a été ratifiée par la Commission européenne en 2009. L'UE manifeste un intérêt réel pour le modèle de gouvernance régionale. La bonne réalisation des programmes de coopération et l'intensité des échanges (de personnes et de marchandises) fait en effet de la Baltique une référence en comparaison d'autres espaces régionaux.

L'institutionnalisation de la coopération en mer Baltique s'est accélérée au début de la décennie 1990. Elle concerne notamment l'ensemble des acteurs du monde maritime : les autorités municipales et portuaires, les acteurs industriels, les transporteurs, les compagnies ferries, les agences de voyage. Le souvenir de la Hanse joue ici un rôle essentiel en tant que premier moment d'unité de la zone. À l'échelle de la région baltique, l'instrumentalisation dès les années 1990 de métaphores comme celle de la " nouvelle Hanse " montre à quel point les flux maritimes ont joué un rôle important dans le projet politique d'unité baltique $^{13}$. Créé en 1980, le réseau "Hanse des temps nouveaux » ou " nouvelle Hanse " est un groupement d'anciennes villes hanséatiques dont le siège se situe à Lübeck. L'espace baltique a vu la création d'instances de coopération parmi lesquelles l'Organisation des ports de la Baltique (BPO), créée en 1991 afin de faciliter la coopération entre les ports. Les missions du réseau sont de plusieurs ordres : fournir des statistiques sur les volumes des trafics de tous les ports, lutter à l'échelle de l'espace baltique contre la pollution et assurer une activité portuaire propre, permettre, enfin, un échange d'expériences et une formation continue des équipes des différents ports.

La reconnexion entre les rives passe par la mise en place d'un système de transport efficace autour de l'organisation d'un réseau maritime pour favoriser la coordination des échanges. Ces initiatives sont propices à l'accroissement du trafic maritime, ce qui entraîne des problèmes d'une part, environnementaux, et d'autre part, de sécurité maritime ${ }^{14}$. Dans le domaine de l'intermodalité, il est possible de citer l'exemple des autoroutes de la mer (Motorways of the sea). Le concept a été développé pour la première fois à partir d'un livre blanc, $\mathrm{La}$

12 Pawel Samecki, Macro-regional strategies in the EU, Discussion Paper presented by Commissioner Pawel Samecki in Stockholm on 18 September 2009, 6 p.

13 Nicolas Escach, «La nouvelle Hanse de Björn Engholm a-t-elle été vraiment un échec? », Eurostudia, 2011, [www.cceae.umontreal.ca/IMG/pdf/Eurostudia_vol.7_-_N._Escach.pdf] (consulté le 3 octobre 2017).

14 Élise Lépy, Les glaces de mer en mer Baltique..., op. cit. 
politique européenne des transports à l'horizon 2010. L'heure des choix, publié par la Commission européenne en 2001. Dans le cadre de plusieurs appels à projets (2008-2009) financés par les réseaux transeuropéens de transport (TEN-T), trois projets ont pu être financés : une amélioration de la liaison fixe entre Klaipeda et Karlshamn et entre Karlskrona et Gdynia et des investissements sur l'intermodalité entre Sassnitz et Trelleborg. Les autoroutes de la mer, portées par l'UE et visant au développement d'un transport durable en favorisant un report modal de la route vers la mer, ne peuvent cependant pas concerner un port russe, ce qui limite de fait leur pertinence dans la région.

L'espace baltique voit une régionalisation progressive se mettre en place dans le cadre des transports ou de l'environnement notamment. Il est désormais défini comme un espace de coopération/compétition né après $1991^{15}$. Par exemple, si la référence à la Hanse est souvent utilisée pour marquer l'unité maritime et portuaire baltique, elle doit être nuancée. Ainsi, le modèle portuaire hanséatique, notamment en termes de gouvernance (ports propriété de la commune dans laquelle ils se sont développés), est loin d'être commun à l'ensemble de la région.

Le concept de la renaissance d'une idée hanséatique est présent dans l'espace baltique et illustre sa structuration régionale. Cependant, les faits montrent à quel point chaque acteur (État, organisme portuaire, compagnie privée) est engagé dans la conquête de marchés pour assurer le développement de son propre espace économique, de sa propre entreprise ou en lien avec des considérations géopolitiques. Aujourd'hui, nous pouvons parfois parler de coopétition ${ }^{16}$. Il s'agit d'une compétition dans laquelle les évolutions sont rapides. Le principe économique du " gagnant-gagnant » qui permettrait à chacun de tirer les fruits de la croissance du marché est largement dépassé par un principe plus simple à comprendre : celui $\mathrm{du}$ " gagnant-perdant».

Enfin, la dimension politique européenne est présente dans la région. Elle se matérialise via les réseaux transeuropéens de transports, les RTE-T, notamment leur pan maritime : les autoroutes de la mer. Définies comme un "réseau de liaisons maritimes entre les États membres ", elles doivent permettre d'exploiter au mieux une caractéristique du transport intracommunautaire maritime : un nombre élevé de liaisons à courte distance à vocation logistique. Ces itinéraires de cabotage soutenus financièrement par l'UE participent à atteindre l'objectif de transfert modal et à assurer une meilleure cohésion territoriale.

15 Nathalie Blanc-Noël, La Baltique. Une nouvelle région en Europe, Paris, L'Harmattan, 2003.

16 Collaboration ou une coopération de circonstance ou d'opportunité entre différents acteurs économiques qui, par ailleurs, sont des concurrents. 


\section{UNE MER À L'INTERFACE ENTRE L'UNION EUROPÉENNE ET LA RUSSIE}

La régionalisation baltique apparaît évidente. Toutefois, comme la Méditerranée, la Baltique maritime reste un espace hétérogène sur lequel ne s'est pas structurée une véritable façade maritime qui serait le miroir d'une économie pourtant fortement tournée vers la mer. Les bouleversements contemporains d'ordres globaux et régionaux redessinent et complexifient la carte de cette interface. Des nœuds nouveaux émergent et ont tendance à centraliser des dynamiques sousrégionales ${ }^{17}$. En effet, si la mer Baltique est un exemple majeur d'entre-deux géographique, l'inscription des ports baltiques dans les réseaux et leur rôle dans la régionalisation differe notamment d'une rive à l'autre et en fonction des liens plus ou moins marqués avec le monde russe.

\section{1. À finalités différentes, ports différents}

Une analyse pointue des trafics portuaires établit que la croissance est en réalité inégalement répartie : cette dernière est en règle générale plus vive en Baltique orientale qu'en Scandinavie (voir fig. 1). Illustrant l'importance de l'acteur russe, le golfe de Finlande qui offre les conditions nautiques parmi les moins favorables de la région (récifs, emprise hivernale des glaces...) concentre l'augmentation du trafic maritime.

En fait, la mer Baltique est le support de cinq types de trafics répondant à des stratégies économiques différentes :

- le trafic de minerais et de matières premières à l'exportation est concentré sur quelques ports spécialisés, majoritairement russes, baltes et polonais. Il dépend de la conjoncture économique internationale ;

- le trafic du bois éclaté sur de nombreux ports (surtout des petits ports suédois et finlandais) est aussi dépendant de centres économiques extérieurs ;

- le trafic des hydrocarbures est un marché captif répondant aux besoins de la Russie et se concentre en Baltique orientale ;

- le trafic de marchandises générales où la filière conteneurisée pèse de plus en plus et entre dans les stratégies des grands opérateurs de transport à l'échelle de l'Europe. S'il se développe dans toute la région, sa croissance est vive dans les ports baltes et russes, ce qui n'est pas le cas dans les ports nordiques dont la situation limite leur rôle d'interface est-ouest ;

- le transport de passagers est marqué par des enjeux importants entre armements qui cherchent à dominer le transport. Étroitement liés aux lignes roulières, il se concentre dans les franchissements des détroits et des bras de mer.

17 Nora Mareï, César Ducruet, "L'intégration économique de la Méditerranée par les réseaux maritimes et portuaires ", Maghreb-Machrek, no 220, 2014/2, p. 11-33. 
Ces stratégies économiques se retrouvent dans l'organisation de l'espace maritime baltique. Des ports nordiques, à vocation essentiellement régionale ou nationale, côtoient des ports moyens allemands dominés par les flux rouliers nord $/$ sud $^{18}$. Les ports polonais desservent essentiellement le territoire national même si Gdansk se positionne désormais dans les réseaux conteneurisés. Côté balte, les ports sont restés et restent, malgré la disparition de l'URSS, la façade maritime de la Russie et de la Biélorussie, voire d'autres États de la CEI. Cette situation est également celle de ports finnois (Hamina/Kotka et dans une moindre mesure Helsinki), même si la plupart ont vocation à desservir le territoire national à fort caractère insulaire.

Enfin, depuis la fin des années 1990, la Russie a mis en place une politique de développement portuaire visant à l'autosuffisance en réduisant sa dépendance à l'égard des ports baltes devenus étrangers. Le premier port de la région est désormais Oust-Louga, port qui n'existait pas avant 2006. La question du trafic russe se pose d'autant plus que les frontières entre la Russie et les Républiques baltes coïncident avec celles de l'Union européenne, ce qui ne facilite la circulation ni des marchandises ni des personnes. Toutefois, profitant de la croissance de la demande, la part du transit n'a pas diminué malgré des variations conjoncturelles en fonction du type de marchandises. D'ailleurs, les intérêts russiens continuent à s'implanter dans les ports des pays baltes ${ }^{19}$. Ainsi, si d'un point de vue général les investisseurs dans les pays baltes sont principalement suédois, allemands, norvégiens, finlandais et danois, ce n'est pas du tout le cas dans le monde portuaire où ils sont plutôt russes, biélorusses ou des acteurs globaux du monde maritime. Parmi les nombreux exemples, nous pouvons citer l'un des leaders russes dans la production d'engrais, la société UralChem qui possède désormais $51 \%$ des actions du nouveau terminal Riga Fertilizer Terminal LLC (RFT) du port de Riga.

Par ailleurs, la recomposition des flux dans la région se matérialise par le développement de nouvelles infrastructures. Les projets et constructions de ports sont nombreux, mais ils sont de plus grande envergure dans les anciens pays socialistes : les plus ambitieux se situent surtout en Russie qui a dû remplacer la capacité des ports soviétiques devenus baltes.

\subsection{Les circulations au cour d'un phénomène de sous-régionalisation : l'exemple du trafic conteneurisé}

Le trafic conteneurisé introduit une nette différenciation des activités maritimes et portuaires au sein de la mer Baltique. Les ports de la Baltique orientale

18 Les portes d'entrée de l'Allemagne sont Hambourg, Bremerhaven, voire Rotterdam ou Anvers, pas les ports de la Baltique.

19 Pierre Thorez, "Les enjeux portuaires de la Russie en mer Baltique ", Territoire en mouvement, $n^{\circ} 10,2011$, p. 49-59. 
comme Saint-Pétersbourg, Klaipeda, Helsinki ou Gdansk ainsi que des ports de l'Øresund comme Göteborg et Aarhus apparaissent comme les pôles majeurs. Ils se modernisent et s'internationalisent au rythme des extensions portuaires et de l'installation d'acteurs économiques internationaux de la logistique et de l'industrie. Outre la modernisation des infrastructures portuaires, la mise en réseau croissante des ports est le signe d'une recomposition des logiques d'acteurs. Sur la rive orientale de la mer Baltique comme dans les détroits danois, la mise en réseau intra-baltique des ports est évidente (voir fig. 2). Le déplacement du centre de gravité de l'espace baltique vers l'est semble une évidence au regard des réseaux maritimes.

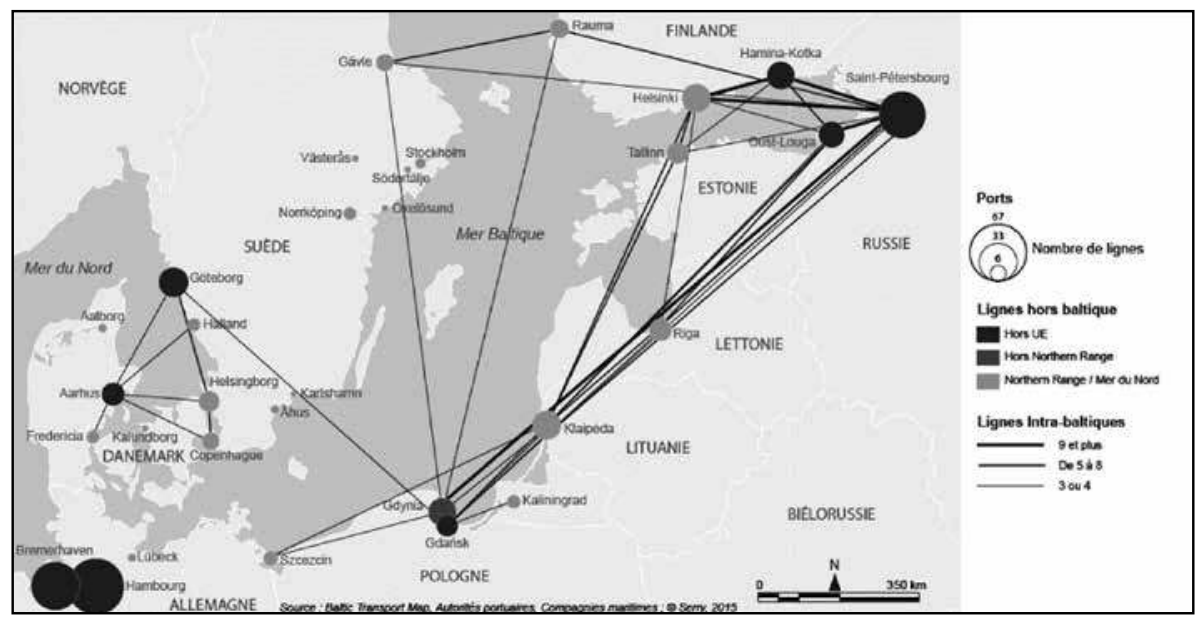

Figure 2 - Les dessertes conteneurisées en mer Baltique en 2015

Le transport maritime de conteneurs est symptomatique de l'insertion des ports dans les lignes régulières maritimes, donc de leur intégration dans le commerce mondial. L'analyse du trafic conteneurisé permet de dresser une typologie des ports à conteneurs baltiques :

- les ports d'importance régionale traditionnels comme Göteborg et Trelleborg dont la localisation et la précoce ouverture aux marchandises conteneurisées expliquent l'importance contemporaine. Ces ports scandinaves entretiennent traditionnellement des liens économiques étroits avec l'Ouest de l'Europe, s'appuyant sur un dense réseau de services maritimes, rouliers ou conteneurisés, à travers et hors de la Baltique ;

- des ports de desserte régionale ou nationale, principalement localisés sur la rive occidentale de la mer Baltique. Le trafic souvent modeste de ces ports ne doit pas minimiser leur rôle dans les économies régionales, surtout pour les régions «lointaines» du golfe de Botnie, par exemple ; 
- les ports de la "Russie " sont eux constitués des ports russes, baltes et finnois (Hamina/Kotka). Les chiffres confirment le rôle majeur du port de SaintPétersbourg dans le trafic conteneurisé, véritable gateway ${ }^{20}$ de la Russie avec près de 2 millions d'EVP en 2015. Cet essor est lié à la mise en service de nouvelles capacités, notamment à Klaipeda avec l'ouverture de la seconde tranche du terminal ou encore à Oust-Louga. Plusieurs ports présentent des combinaisons semblables, exploitant au moins partiellement le même arrière-pays.

Le rôle des ports de la Baltique orientale reste primordial dans la desserte de la Russie, notamment pour l'acheminement des conteneurs vers les zones de consommation : le cœur économique de la Russie (région de Moscou) et quelques destinations plus lointaines. L'héritage soviétique vient renforcer le particularisme de ce système de transport de marchandises, car la plus grande part du fret international entre Baltique et ces régions est constituée de fret ferroviaire. Une typologie fonctionnelle des ports régionaux se dégage ainsi : au nord et à l'ouest des ports de marché nationaux ; au sud, avec Klaipeda et surtout Gdansk, des ports s'affirment comme intermédiaires ( $h u b s$ ) régionaux, intermédiaires entre la Baltique et les grandes routes maritimes; à l'est, les ports sont des interfaces entre le monde russe et l'espace européen.

\subsection{Le jeu des tensions géopolitiques}

Les relations entre la Russie et les États baltes n'ont jamais été sereines. Durant toutes les années 1990, les presses baltes et russes se sont fait l'écho de ces tensions, des pressions et menaces de sanction. "Moscou et Riga préparent le blocus " titrent les Izvestia du 11 mars 1998 quand la Russie envisage des sanctions économiques contre la Lettonie pour sa législation restrictive à l'égard des russophones $^{21}$. Mais le journal ne manque pas de souligner que c'est une arme à double tranchant, car dans le même temps, une partie de ces " russophones " sont actifs dans le commerce local et les ports. Pour sortir de la dépendance, les autorités russes ont très tôt développé leurs propres infrastructures portuaires en modernisant les deux grands ports historiques de Saint-Pétersbourg et Kaliningrad. Leurs limites, encombrement et durée de la période de gel pour le premier, enclavement pour le second ont reporté la stratégie vers des sortes d'avant-ports à 70-90 kilomètres à l'ouest de Saint-Pétersbourg, aux entrées nord et sud du golfe de Finlande. Cela est symbolisé par le chantier du terminal de Primorsk inauguré en 2001 par Poutine qui marque un tournant dans les capacités russes d'exportation d'hydrocarbures, affaiblissant le rôle que jouaient jusqu'alors Ventspils,

20 Un gateway est un espace polarisant des flux très importants avec un impact sur le territoire environnant. Les marchandises sont diffusées à partir de cette région gateway dans un milieu différent et avec des modes différents.

21 Jean Radvanyi, la façade balte, Annuaire de l'Observatoire franco-russe auprès de la chambre de commerce franco-russe, 2017. 
Tallinn et Klaipeda. Désormais, c'est sur la rive sud du golfe - elle bénéficie d'accès plus facile aux régions - qu'on assiste aux développements les plus spectaculaires, principalement à Oust-Louga.

Tendues depuis l'éclatement de l'URSS en 1991, les relations se sont encore détériorées en 2016. L’UE et les États-Unis imposent des sanctions économiques à la Russie depuis juillet 2014. La Russie a répondu par un embargo sur les produits agro-alimentaires occidentaux et Moscou a profité des sanctions pour adopter une stratégie de substitution : frapper les importations pour inciter à une production nationale. De ce fait, depuis 2014, la concurrence se renforce. En effet, cette façade maritime est désormais soumise à des conditions économiques changeantes : la stagnation de la demande russe et les sanctions économiques européennes à l'égard de la Russie et stratégies des alliances maritimes remettent en cause l'augmentation globale de la demande et la croissance partagée au profit d'une compétition accrue. Le recul des trafics dans certains ports russes et dans les ports baltes comme Tallinn et Ventspils sont révélateurs de cette évolution : l'hyperdépendance à l'égard de la Russie est un facteur de fragilité des ports de la Baltique orientale. Ainsi l'analyste économique letton Aleksandr Butskovski estime que la situation s'est aggravée pour des raisons politiques ${ }^{22}:$ «Au début, les sanctions économiques réciproques de l'Union européenne et de la Russie n’ont pas exercé d'impact notable sur le fonctionnement des ports lituaniens, lettons et estoniens, mais la décision de Moscou de cesser, d'ici 2018, l'exportation de ses produits pétroliers par les ports des pays baltes peut changer complètement la donne."

La conjoncture du commerce international, qui connaît une baisse relative depuis 2013, complexifie la lecture. Il est difficile de distinguer dans les fluctuations de trafic ce qui tient à cette conjoncture et ce qui découle de décisions politiques d'inverser les flux. Même si le volume du commerce entre la Russie et l'UE a subi à la fois les effets des sanctions et contre-sanctions et la baisse des cours de matières premières pour atteindre un niveau particulièrement bas (le volume total import + export est ainsi passé de 417 milliards de dollars en 2013 à 235 en 2015 et seulement 201 en 2016 !), la Russie demeurait, en 2015, un des trois principaux partenaires commerciaux de la Lettonie et de la Lituanie. Si la situation est tendue pour les ports baltes, elle peut également impacter l'économie russe même si en Russie, des médias se sont réjouis de l'espèce de boycott annoncé sur le transit par les ports baltes, mêlant parfois des considérations économiques à un certain esprit de revanche. En effet de nombreuses sociétés russes, tant locales que relayant des capitaux de Russie, ont pris des parts importantes dans divers secteurs économiques. Ces tensions n’auront-t-elles pas également des conséquences

22 [https://fr.sputniknews.com/economie/201609301027999529-russie-ports-lettonie-estonielituanie-exportation-petrole-refus/(consulté le 15 septembre 2017). 
négatives sur les intérêts russes sur place ? Ils sont difficiles à évaluer et font l'objet de polémiques, tant sur leur volume que sur leurs objectifs (on parle souvent à Riga et Tallinn de " cinquième colonne $»^{23}$ ).

La montée des tensions dans la région reste toutefois défavorable aux échanges. C'est pourquoi les ports les mieux intégrés dans les réseaux maritimes, mais aussi les plus diversifiés en termes de marchandise et d'arrière-pays, sont ceux qui s'en sortent désormais le mieux : c'est le cas de Gdansk ou Klaipeda (voir fig. 2). La majorité des ports de la façade orientale gardent cette vocation d'interface même si certains connaissent des difficultés d'adaptation en raison de leur localisation ou de leur hyperdépendance au transit russe. Enfin, des enjeux se font jour à l'échelle eurasiatique : capter une partie des flux engendrés par le programme transcontinental chinois de la nouvelle Route de la soie comme l'illustre la signature en juin 2015 d'un accord entre la compagnie KTZ Express, filiale des chemins de fer kazakhes, et le port lituanien de Klaipeda.

\section{Des DYNAMIQUeS RÉCENTES FaVORABLES À LA CONCURRENCE ET À LA DIFFÉRENCIATION}

La diversité maritime et portuaire baltique prend tout son sens au regard de la multiplicité des situations. Après avoir appréhendé cette question principalement sous l'angle portuaire, une approche par les marchandises, les flux et leurs organisateurs renforce sa compréhension. En réalité, face aux intérêts russes, les autres pays riverains de la Baltique semblent agir en ordre dispersé alors que l'Union européenne ne perçoit pas cet espace comme stratégique ${ }^{24}$.

\subsection{Des flux énergétiques révélateurs des différenciations}

La question des circulations énergétiques est primordiale dans la région tant au point de vue économique que politique. Celle-ci a souvent symbolisé les évolutions et exacerbé les tensions. Le gazoduc Nordstream ${ }^{25}$ inauguré en 2011, reliant Portovaïa (près de Vyborg) à Greifswald en Allemagne, illustre cette volonté russe de s'émanciper des ports de la Baltique. Par ailleurs, en septembre 2015, malgré la crise ukrainienne, un protocole a été signé pour la construction d'un second tube, contesté par plusieurs pays de l'UE.

Le volume des exportations russes a fortement reculé depuis 2013, mais le pétrole en représente encore à lui seul $54 \%$. Les hydrocarbures russes occupent

23 Jean Radvanyi, op. cit.

24 Jean-François Fiorina, "Géopolitique de la Baltique, un espace entre concurrence et coopération », Comprendre les enjeux stratégiques, no 110, 27 juin 2013.

25 Compagnie mixte présidée par l'ancien chancelier allemand Gerhard Schröder (Gazprom y détient $51 \%$ des actions, Wintershall et EON 15,5\%, Nederlandse et GDF $9 \%$ ). 
donc une place prépondérante dans les flux à l'exportation, principalement dans les ports de la Baltique orientale. Les hydrocarbures, et tout particulièrement le pétrole brut et le gaz naturel, représentent un enjeu particulier en raison des fortes relations d'interdépendance existant entre l'Union européenne et la Russie dans ce domaine. La Russie a besoin de la Baltique pour exporter ses hydrocarbures, dont les volumes exportés sont en constante et rapide augmentation. En effet, son accès à l'océan mondial est limité par la spécialisation portuaire héritée de l'époque soviétique et la perte d'une partie de son accès à la mer liée à la disparition de l'URSS. Elle a donc été obligée d'utiliser, presque uniquement, les ports baltes pour ses exportations jusqu'au début des années 2000 .

Comme d'autres trafics, après avoir profité aux ports baltes, celui-ci s'est réorienté vers les ports russes, principalement Primorsk qui concentre 75 millions de tonnes de fret pétrolier, soit $25 \%$ du total des exportations russes de pétrole, et le port d'Oust-Louga. En effet, la Russie a opéré un resserrement progressif des terminaux d'exportation vers le territoire national. Les ports baltes (Tallinn, Ventspils et dans une moindre mesure Klaipeda) ont de plus en plus de difficultés à capter ces trafics, car le pouvoir politique russe ne cesse d'œuvrer pour parvenir à cette autosuffisance portuaire. Par la relance de ses ports, la Russie compte conserver, voire renforcer son rôle dans cet espace baltique. Désormais, même si des intérêts russiens persistent dans les ports baltes, comme l'entreprise pétrolière Lukoil qui, en 2010, a participé à la modernisation du terminal pétrolier de Ventspils, cette situation est une réalité : le pétrole russe est désormais en grande majorité exporté par les ports russes de la mer Baltique.

Le système de transport des hydrocarbures russes a donc glissé d'une complémentarité imposée par les héritages historiques à une concurrence exacerbée et à une différenciation dans le rôle des ports. D'autant plus que, si les flux d'hydrocarbures constituent par ailleurs des trafics importants dans certains ports scandinaves (Brofjorden, Sköldvik...), c'est principalement à l'importation pour répondre aux besoins de la Suède et de la Finlande. Il y a donc une évidente dichotomie entre des ports nordiques qui sont des ports de marché, principalement d'importation, supportant des flux ouest-est et des ports russes et baltes à vocation mondiale exportant des flux de pétrole d'est en ouest.

Par ailleurs, le cas du gaz naturel liquéfié (GNL), au cœur de nouvelles dynamiques de transition, est intéressant à double titre. En effet, il s'agit d'une marchandise nouvelle dans les ports de la région qui dépasse les seuls intérêts économiques et qui revêt une dimension géopolitique. Le gaz naturel est une source importante d'énergie en Europe et dans la région de la mer Baltique en particulier. La logistique du gaz naturel en Europe est aujourd'hui largement tributaire 
des connexions aux réseaux de gazoducs. Cette géographie des conduites explique d'ailleurs en partie la dépendance balte à l'égard des livraisons gazières russes.

Deux grands pilotes guident le développement des installations de GNL en Baltique. Tout d'abord, le GNL est considéré comme un moyen de sortir de la dépendance énergétique à l'égard de la Russie par la diversification des approvisionnements en gaz et en permettant plus de souplesse ${ }^{26}$. La seconde justification de cet intérêt accru pour le GNL en mer Baltique est sa capacité à servir comme combustible marin conforme aux dernières réglementations.

Les ports de la baltique orientale montrent un intérêt accru pour les installations de gaz naturel liquéfié (GNL). Le premier terminal à Klaipeda est devenu opérationnel fin 2014. Ce terminal flottant baptisé Indépendance a pour objectif de diminuer la dépendance énergétique à l'égard de la Russie et d'accroître la diversification de l'approvisionnement en gaz de la Lituanie et de ses voisins lettons et polonais. Les crises énergétiques récurrentes entre l'UE et la Russie ont particulièrement pesé sur cette volonté de diversification.

Enfin, la question des flux de GNL pose de nouveau la question de la coopétition et dépasse le simple cadre maritime comme l'illustre le cas de Nord Stream 2, projet de doublement du gazoduc Nord Stream qui, depuis 2012, relie la Russie à l'Allemagne via la mer Baltique. Courant sous la mer Baltique sur 1220 kilomètres, doté comme le Nord Stream 1 d'une capacité de 55 milliards de $\mathrm{m}^{3}$ de gaz par an, le Nord Stream 2 vient de voir signer un accord de financement entre les entreprises Wintershall, Uniper (Allemagne), Royal Dutch Shell (Royaume-Uni/ Pays-Bas), OMV (Autriche) et Engie (France). Il devrait voir sa construction lancée en 2018 et s'achever, officiellement, fin $2019^{27}$.

\subsection{Des jeux d'acteurs originaux}

Qu'il s'agisse de marchandises atypiques ou de stratégies d'acteurs maritimes comme les armateurs, multiples sont les exemples de concurrence et/ou de complémentarité entre ports et acteurs économiques des pays riverains - ou à proximité - de l'espace baltique.

De nombreux producteurs d'engrais azotés utilisant le gaz naturel russe comme matière première sont localisés dans l'Ouest de la Russie, de la Biélorussie et en Lituanie. Les exportations d'engrais chimiques sont très développées dans l'Est de

26 Kari Liuhto, "Liquefied Natural Gas in the Baltic Sea Region ", Journal of East-West Business, no 19(1-2), 2013, p. 33-46.

27 Céline Bayou, «Russie : Fort de son accord de financement, le gazoduc Nord Stream 2 cherche sa route ", in Regard sur l'Est, 20 mai 2017, [http://www.regard-est.com/home/breves.php?idp=1931] (consulté le 3 octobre 2017). 
la Baltique, constituant par exemple la majorité des produits chimiques manutentionnés dans les ports lituaniens (30\% du trafic total). Les engrais représentent donc un élément clé dans le trafic de vrac sec régional ${ }^{28}$.

Environ $80 \%$ des exportations d'engrais minéral russe passent par les ports de la mer Baltique : les ports de la Baltique orientale servent de plateformes de distribution vers le marché mondial. Si les flux d'engrais sont volatiles, comme les produits agricoles, dépendants de contraintes exogènes, quelques tendances et persistances régionales se dégagent.

Dans la région, l'intégration verticale de la filière " engrais " s'est renforcée au cours des dernières années. De plus en plus de producteurs d'engrais chimiques se positionnent en effet dans la chaîne logistique de leurs produits. L'exemple lituanien est particulièrement parlant : le port de Klaipeda est le lieu privilégié d'exportation des engrais biélorusses. L'intégration précédemment évoquée y est à l'œuvre comme l'illustre la prise de participation dans un terminal combiné BKT dédié aux engrais du principal fabricant biélorusse, la société Belaruskali. BKT va construire deux nouveaux entrepôts de 40000 tonnes de capacité, ce qui devrait encore renforcer le leadership de Klaipeda.

Autre manifestation concrète de cette situation, l'un des leaders russes dans la production d'engrais, la société UralChem possède désormais $51 \%$ des actions du nouveau terminal Riga Fertilizer Terminal LLC (RFT) du port de Riga. Ce terminal, dont la construction a coûté 62 millions d'euros, a déjà manutentionné 2 millions de tonnes d'engrais depuis son ouverture en février 2014. UralChem prévoit d'en utiliser environ la moitié pour du nitrate d'ammonium.

Il y a deux principaux fournisseurs de potasse sur le territoire post-soviétique : le russe Uralkali et le biélorusse Belaruskali. Jusqu'en 2013, les entreprises suivaient une politique de vente concertée, avec une majorité de leurs exportations réalisées par leur joint-venture, Belarusian Potash Company. Cependant, les entreprises ont cessé leur collaboration et ont commencé à remodeler leur logistique export.

Les jeux d'acteurs dans la région sont multiples, le conflit entre la compagnie russe Uralkalij et Belaruskalij a ainsi eu des conséquences sur le trafic du port de Klaipeda en 2013 et explique en partie la baisse du trafic cette année-là, puis sa reprise dans une période récente.

D'un autre point de vue, les comportements des transporteurs maritimes sont à la fois des révélateurs du dynamisme portuaire et des moteurs de ce dernier : leurs choix impactent directement les possibilités de développement des ports.

28 Arnaud Serry, "Mer Baltique : à l'écart des flux agricoles, mais au cœur de la circulation des engrais ", Le DEMETER, 2016, p. 311-323. 
En Baltique, en 2015, vingt-huit compagnies (hors alliances) étaient présentes sur le marché conteneurisé. Parmi elles, des compagnies importantes (Unifeeder, basée à Århus, Team Lines, basée à Hambourg) et des compagnies de niveau mondial comme MSC ou CMA-CGM qui sont très bien implantées. Plusieurs compagnies régionales complètent l'offre comme Seagoline (Groupe A.P. Moller - Maersk), Containerships (Finlande), Mannlines (Allemagne), Tschudi Line (Norvège) ou Sea Connect (Lituanie). Le marché conteneurisé se caractérisait ainsi par une forte densité de compagnies maritimes, qui desservaient les ports de la région à partir des ports nord-européens. Les ports baltiques ne constituent donc pas des relais des grands flux mondiaux et européens, mais plutôt un espace secondaire d'échanges.

Le dynamisme du marché, malgré la crise en Russie, attire régulièrement de nouveaux acteurs. Ainsi, de novembre 2015 à novembre 2016, soixante opérateurs différents ont proposé des services conteneurisés en mer Baltique. Les comportements de ces opérateurs peuvent être identifiés : les leaders mondiaux (MAERSK, MSC ou CMA CGM) concentrent leurs trafics sur un nombre réduit de ports alors que les compagnies spécialisées dans le feedering (Unifeeder) ont un réseau décentralisé.

Les ports sont donc fortement dépendants des stratégies : le cas de Gdansk est intéressant car, en dépit du faible nombre de lignes, son trafic est relativement élevé en raison de son rôle de port de transbordement régional comme il est desservi par des navires de plus grande taille (4 lignes hebdomadaires le relient d'ailleurs à des ports hors Europe) dans le cadre de l'alliance intervenue en avril 2017 entre MAERSK et MSC.

Dans les faits, les investissements se multiplient dans les ports de la Baltique orientale et intègrent désormais la dimension logistique. Par exemple, la compagnie finlandaise SRV a lancé un centre logistique de classe A de 120000 mètres carrés à Saint-Pétersbourg. Le développement de ces fonctions logistiques se généralise à proximité de la plupart des ports à conteneurs de la région plaçant ces ports au cœur des réseaux et des systèmes de distribution.

Dans le même ordre d'idées, les stratégies des acteurs maritimes charpentent le réseau roulier si présent en mer Baltique et souvent considéré comme spatialement structurant à des échelles locales (dans les détroits danois, par exemple) ou dans la mise en relation des différentes rives.

Les jeux d'acteurs autour des circulations énergétiques ou de la mise en place de réseaux maritimes efficients dans la région rappellent le rôle de couture de la mer Baltique par son importance régulièrement croissante dans des relations 
économiques de plus en plus globalisées. Toutefois, des éléments de coupure apparaissent à la fois au sein de l'espace Baltique via l'insertion (ou non) des ports dans les réseaux maritimes et entre la Baltique et le monde russe. Dans ce double jeu, acteurs économiques et politiques semblent avoir des comportements quelques peu différents : face au discours très partial des acteurs publics, les entreprises privées font preuve d'une approche plus pragmatique.

\section{Conclusion}

La mer comme support d'échanges maritimes constitue indéniablement un trait d'union entre pays de l'espace baltique, où l'activité maritime et portuaire connait depuis une vingtaine d'années un dynamisme certain. La prise en compte du fait maritime, de son importance économique parfois vitale pour les économies locales, mais aussi de ses impacts constitue ainsi un dénominateur commun sur les rives de la Baltique. Quoi qu'il en soit, parler d'homogénéité baltique semble hâtif, voire inapproprié. Si globalement, par son intermédiarité, le système maritime et portuaire baltique se caractérise par son rôle d'interface, les disparités entre les rives, voire entre les ports eux-mêmes et les dynamiques qui leurs sont liées rappellent la diversité et la complexité du dit système.

En effet, qu'il s'agisse des types de trafics, des modes de gouvernance des ports, de leurs fonctions (port de transit, transbordement), de leurs poids dans les économies locales, les différences entre les ports de la région sont multiples. Le rapprochement entre les rives se fait moins par les circulations entre les rives que par leur insertion dans des réseaux structurés autour des pôles majeurs du transport maritime mondial. Si des sous-régionalisations sont pertinentes : détroits danois, rangée portuaire orientale ou encore ports du Sud de la Baltique, la recomposition portuaire contemporaine dans la région renforce plutôt le positionnement des ports dans le contexte du commerce maritime mondialisé, que dans celui d'une intégration régionale portée par la mer. L'activité maritime et portuaire dans l'espace restreint de la Baltique est marquée par la concurrence plus que par la complémentarité, par la multiscalarité plutôt que par l'homogénéité régionale. Ainsi, les ports baltes, de taille relativement importante dans le cadre baltique, fortement concernés par le transit de matières premières russes sont fort différents des ports nordiques ou suédois, de taille modeste et tournés vers leur marché national. Les circulations marquées par l'omniprésence russe, notamment dans les flux énergétiques, apparaissent comme susceptibles de peser fortement sur les dynamiques économiques régionales tout comme sur la diplomatie régionale et la géopolitique à cette frontière orientale entre l'Union européenne et la Fédération de Russie. 
Si l'espace baltique reste un espace de coopération maritime, la compétition et la concurrence s'y sont rapidement développées, surtout récemment avec la rétraction du marché et les tensions entre la Russie et les pays occidentaux. Si le monde maritime et portuaire n'est pas isolé du politique, son étude montre que les relations économiques sont moins affectées que d'autres, en raison des besoins qui subsistent et d'un système très intégré qui les satisfait. Toutefois, une détérioration de la situation politique ne manquerait pas d'avoir un effet sur les circulations régionales.

Enfin, l'espace baltique porte les marques de son statut de périphérie intégrée, par la puissance relative de ses besoins, pourtant singulièrement contraints par l'éparpillement et le faible nombre de leurs forces vives ; les acteurs du transport maritime ont adopté des stratégies d'efficience qui ne sont pas sans conséquence sur l'organisation et l'évolution des territoires. 